\title{
Splines for Diffeomorphic Image Regression
}

\author{
Nikhil Singh and Marc Niethammer \\ University of North Carolina, Chapel Hill, USA
}

\begin{abstract}
This paper develops a method for splines on diffeomorphisms for image regression. In contrast to previously proposed methods to capture image changes over time, such as geodesic regression, the method can capture more complex spatio-temporal deformations. In particular, it is a first step towards capturing periodic motions for example of the heart or the lung. Starting from a variational formulation of splines the proposed approach allows for the use of temporal control points to control spline behavior. This necessitates the development of a shooting formulation for splines. Experimental results are shown for synthetic and real data. The performance of the method is compared to geodesic regression.
\end{abstract}

\section{Introduction}

With the now common availability of longitudinal and time-series image data, models for their analysis are critically needed. In particular, spatial correspondences need to be established through image registration for many medical image analysis tasks. While this can be accomplished by pair-wise image registration to a template image, such an approach neglects spatio-temporal data aspects. Instead, explicitly accounting for spatial and temporal dependencies is desirable.

Methods that generalize Euclidean parametric regression models to manifolds have proven to be effective for modeling the dynamics of changes represented in time-series of medical images. For instance, methods of geodesic image regression [6] and longitudinal models on images [10 generalize linear and hierarchical linear models, respectively. Although the idea of polynomials [5] and splines [11] on landmark representation of shapes have been proposed, these higher-order extensions for image regression remain deficient. While Hinkle et al. [5] develop general polynomial regression and demonstrate it on finitedimensional Lie groups, the infinite dimensional regression is demonstrated only for the first-order geodesic image regression.

Contribution. We propose: (a) a shooting based solution to cubic image regression in the large deformation (LDDMM) setting, (b) a method of shooting cubic splines as smooth curves to fit complicated shape trends while keeping data-independent (finite and few) parameters, and (c) a numerically practical algorithm for regression of "non-geodesic" medical imaging data. This article is structured as follows: $\S 2$ reviews the variational approach to splines in Euclidean space and motivate its shooting formulation for parametric regression. $\S 3$ then generalizes this concept of shooting splines for diffeomorphic image regression. We discuss experimental results in $\S$ 田

P. Golland et al. (Eds.): MICCAI 2014, Part II, LNCS 8674, pp. 121-129, 2014.

(C) Springer International Publishing Switzerland 2014 


\section{Shooting-Splines in the Euclidean Case}

Variational Formulation. An acceleration controlled curve with timedependent states, $\left(x_{1}, x_{2}, x_{3}\right)$ such that, $x_{2}=\dot{x}_{1}$ and $x_{3}=\dot{x}_{2}$, defines a cubic curve in Euclidean spaces. This curve minimizes an energy of the form, $E=\frac{1}{2} \int_{0}^{1}\left\|x_{3}\right\|^{2} d t$, and solves an underlying constrained optimization problem,

$$
\underset{x_{1}, x_{2}, x_{3}}{\operatorname{minimize}} \quad E\left(x_{3}\right) \quad \text { subject to } \quad x_{2}=\dot{x}_{1} \text { and } x_{3}=\dot{x}_{2} .
$$

Here $x_{3}$ is referred to as the control variable that describes the acceleration of the dynamics in this system. The unconstrained Lagrangian for the above is,

$$
E\left(x_{1}, x_{2}, x_{3}, \mu_{1}, \mu_{2}\right)=\frac{1}{2} \int_{0}^{1}\left\|x_{3}\right\|^{2} d t+\int_{0}^{1} \mu_{1}^{T}\left(\dot{x}_{1}-x_{2}\right) d t+\int_{0}^{1} \mu_{2}^{T}\left(\dot{x}_{2}-x_{3}\right) d t,
$$

where $\mu_{1}$ and $\mu_{2}$ are the time-dependent Lagrangian variables or the adjoint variables (also called duals) that enforce the dynamic constraints. Optimality conditions on the gradients of the above Lagrangian with respect to the states, $\left(x_{1}, x_{2}, x_{3}\right)$, result in the adjoint system of equations, $\dot{\mu}_{1}=0$ and $\dot{x}_{3}=-\mu_{1}\left(\mu_{2}\right.$ gets eliminated). This allows for a relaxation solution to Eq. (11).

From Relaxation to Shooting. We write the shooting formulation by explicitly adding the evolution of $x_{3}$, obtained by solving the relaxation problem, as a dynamical constraint. This increases the order of the dynamics. Denoting, $x_{4}=-\mu_{1}$, results in the classical system of equations for shooting cubic curves,

$$
\dot{x}_{1}=x_{2}(t), \quad \dot{x}_{2}=x_{3}(t), \quad \dot{x}_{3}=x_{4}(t), \quad \dot{x}_{4}=0 .
$$

The states, $\left(x_{1}, x_{2}, x_{3}, x_{4}\right)$, at all times, are entirely determined by their initial values $\left(x_{1}^{0}, x_{2}^{0}, x_{3}^{0}, x_{4}^{0}\right)$, and in particular, $x_{1}(t)=x_{1}^{0}+x_{2}^{0} t+\frac{x_{3}^{0}}{2} t^{2}+\frac{x_{4}^{0}}{6} t^{3}$.

Shooting-Splines with Data-Independent Controls for Regression. We now present our proposed method of regression using cubic splines using the shooting equations. The goal is to define a smooth curve that best fits the data in the least-squares sense. Since a cubic polynomial by itself is restricted to only fit "cubic-like" data, we propose to add flexibility to the curve by piecing together piecewise cubic polynomials. In other words, we define controls at pre-decided locations in time where the state $x_{4}$ is allowed to jump.

Let, $y_{i}$, for $i=1 \ldots N$, denote $N$ measurements at timepoints, $t_{i} \in(0,1)$. Let $t_{c} \in(0,1)$, for $c=1 \ldots C$, denote $C$ data-independent fixed control locations. For notational convenience, we assume there are no measurements at the end points, $\{0,1\}$, or at the control locations, $\left\{t_{c}\right\}$. The control locations also implicitly define $C+1$ intervals or partitions in $(0,1)$. Let us denote these intervals as $\mathcal{I}_{c}$, for $c=1 \ldots(C+1)$. The constrained energy minimization that solves the regression problem with such a data configuration, 

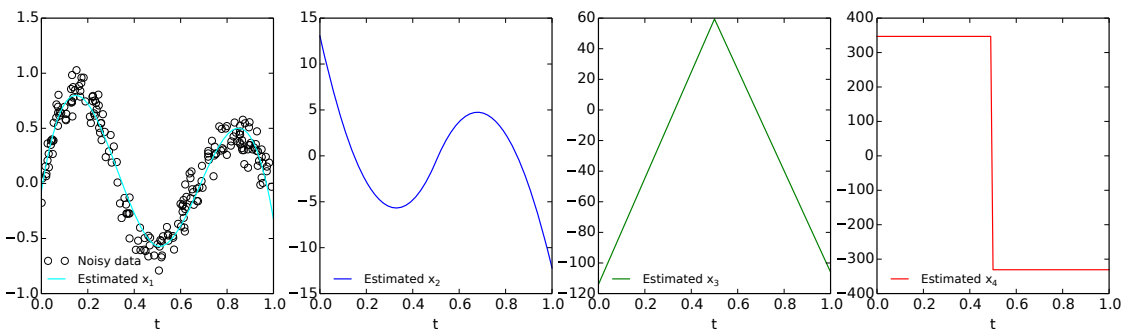

Fig. 1. States for splines regression in Euclidean space with one control at $t=0.5$

$$
\underset{x_{1}(0), x_{2}(0), x_{3}(0), x_{4}(0),\left\{x_{4}\left(t_{c}\right)\right\}}{\operatorname{minimize}} \quad \frac{1}{2 \sigma^{2}} \sum_{c=1}^{C+1} \sum_{i \in \mathcal{I}_{c}}\left\|x_{1}\left(t_{i}\right)-y_{i}\right\|^{2}
$$

s.t. $\quad \dot{x}_{1}=x_{2}(t), \dot{x}_{2}=x_{3}(t), \dot{x}_{3}=x_{4}(t), \dot{x}_{4}=0$,(within each interval, $\left.\mathcal{I}_{c}\right)$, and s.t. $x_{1}, x_{2}$, and $x_{3}$ are continuous across $C$.

The unconstrained Lagrangian enforcing shooting and continuity constraints using time-dependent adjoint states, $\left(\lambda_{1}, \lambda_{2}, \lambda_{3}, \lambda_{4}\right)$, and duals, $\left(\nu_{1}, \nu_{2}, \nu_{3}\right)$, is

$$
\begin{aligned}
E\left(x_{1}^{0}, x_{2}^{0},\right. & \left.x_{3}^{0}, x_{4}^{0}, x_{4}^{t_{c}}, \lambda_{1}, \lambda_{2}, \lambda_{3}, \lambda_{4}\right)=\frac{1}{2 \sigma^{2}} \sum_{c=1}^{C+1} \sum_{i \in \mathcal{I}_{c}}\left\|x_{1}\left(t_{i}\right)-y_{i}\right\|^{2} \\
& +\int_{0}^{1}\left(\lambda_{1}^{T}\left(\dot{x}_{1}-x_{2}\right)+\lambda_{2}^{T}\left(\dot{x}_{2}-x_{3}\right)+\lambda_{3}^{T}\left(\dot{x}_{3}-x_{4}\right)+\lambda_{4}^{T} \dot{x}_{4}\right) d t \\
& +\nu_{1}\left(x_{1}^{-}\left(t_{c}\right)-x_{1}^{+}\left(t_{c}\right)\right)+\nu_{2}\left(x_{2}^{-}\left(t_{c}\right)-x_{2}^{+}\left(t_{c}\right)\right)+\nu_{3}\left(x_{3}^{-}\left(t_{c}\right)-x_{3}^{+}\left(t_{c}\right)\right) .
\end{aligned}
$$

The conditions of optimality on the gradients of the above Lagrangian result in the adjoint system of equations, $\dot{\lambda}_{1}=0, \dot{\lambda}_{2}=-\lambda_{1}, \dot{\lambda}_{3}=-\lambda_{2}, \dot{\lambda}_{4}=-\lambda_{3}$. The gradients with respect to the initial conditions for states $x_{l}^{0}$ for $l=1, \ldots, 4$ are, $\delta_{x_{1}^{0}} E=-\lambda_{1}(0), \delta_{x_{2}^{0}} E=-\lambda_{2}(0), \delta_{x_{3}^{0}} E=-\lambda_{3}(0)$ and $\delta_{x_{4}^{0}} E=-\lambda_{4}(0)$. The jerks at controls, $x_{4}^{t_{c}}$, are updated using, $\delta_{x_{4}^{t_{c}}} E=-\lambda_{4}\left(t_{c}\right)$. The values of adjoint variables required in these gradients are computed by integrating backward the adjoint system. Note that $\lambda_{1}, \lambda_{2}$ and $\lambda_{3}$ are continuous at joins, but $\lambda_{1}$ jumps at the data-point location as per, $\lambda_{1}\left(t_{i}^{+}\right)-\lambda_{1}\left(t_{i}^{-}\right)=\frac{1}{\sigma^{2}}\left(x_{1}\left(t_{i}\right)-y_{i}\right)$. During backward integration, $\lambda_{4}$ starts from zero at each interval at $t_{c+1}$ and the accumulated value at $t_{c}$ is used for the gradient update of $x_{4}\left(t_{c}\right)$.

It is critical to note that, along the time, $t$, such a formulation guarantees that: (a) $x_{4}(t)$ is piecewise constant, (b) $x_{3}(t)$ is piecewise linear, (c) $x_{2}(t)$ is piecewise quadratic, and $(\mathrm{d}) x_{1}(t)$ is piecewise cubic. Thus, this results in a cubic-spline curve. Fig. 1 demonstrates this shooting spline fitting on scalar data. While it is not possible to explain this data with a simple cubic curve alone, it suffices to allow one control location to recover the meaningful underlying trend. The state, $x_{4}$, experiences a jump at the control location that integrates up thrice to give a $C^{2}$-continuous evolution for the state, $x_{1}$. 


\section{Shooting-Splines for Diffeomorphisms}

Notations and Preliminaries. We denote the group of diffeomorphisms by $G$ and its elements by $g$; the tangent space at $g$ by $T_{g} G$; and the Lie algebra, $T_{e} G$, by $\mathfrak{g}$. Let $\Omega$ be the coordinate space of the image, $I$. A diffeomorphism, $g(t)$, is constructed by integrating an ordinary differential equation (ODE) on $\Omega$ defined via a smooth, time-indexed velocity field, $v(t)$. The deformation of an image $I$ by $g$ is defined as the action of the diffeomorphism, given by $g \cdot I=I \circ g^{-1}$. The choice of a self-adjoint differential operator, $L$, determines the right-invariant Riemannian structure on the collection of velocity fields with the norm defined as, $\|v\|_{\mathfrak{g}}^{2}=\int_{\Omega}(L v(x), v(x)) d x$. The velocity, $v \in \mathfrak{g}$, maps to its dual deformation momenta, $m \in \mathfrak{g}^{*}$, via the operator $L$ such that $m=L v$ and $v=K \star m$. The operator $K: \mathfrak{g}^{*} \rightarrow \mathfrak{g}$ denotes the inverse of $L$. For a thorough review of the Riemannian structure on the group of diffeomorphisms, please refer to [12113].

Variational Formulation. As an end-point problem, a Riemannian cubic spline is defined by the curve $g(t)$, that minimizes an energy of the form, $E(g)=$ $\frac{1}{2} \int_{0}^{1}\left\|\nabla_{\dot{g}} \dot{g}\right\|_{T_{g} G}^{2} d t$, where $\nabla$ denotes the Levi-Civita connection and $\|\cdot\|_{T_{g} G}$ is the metric on the manifold at $g$. The quantity, $\nabla_{\dot{g}} \dot{g}$, is the generalization of the idea of acceleration to Riemannian manifolds [7/3. Another way to define the spline is by defining a time dependent control that forces the curve $g(t)$ to deviate from being a geodesic [11. Such a control or a forcing variable, $u(t)$, takes the form, $\nabla_{\dot{g}(t)} \dot{g}(t)=u(t)$. Notice, $u(t)=0$ implies that $g(t)$ is a geodesic.

Taking this idea forward to the group of diffeomorphisms, $G$, we propose to include a time-dependent forcing term that describes how much the 'geodesic requirement' deviates. Thus, we define the control directly on the known momenta EPDiff evolution equation for geodesics 13 , obtained using the right invariant metric to give the evolution in Lie algebra, $\mathfrak{g}$, as $\dot{m}+\mathrm{ad}_{v}^{*} m=0$. Here, the operator $\mathrm{ad}^{*}$ is the adjoint of the Jacobi-Lie bracket 213]. After adding this control, the dynamics take the form, $\dot{m}+\operatorname{ad}_{v}^{*} m=u$, where $u \in \mathfrak{g}^{*}$. Thus we allow the geodesic to deviate from satisfying the EPDiff constraints and constrain it to minimize an energy of the form, $E=\frac{1}{2} \int_{0}^{1}\|u(t)\|_{\mathfrak{g}}^{2} d t$. It is important to note that such a formulation will avoid direct computation of curvature. In other words, since the Levi-Civita connection is expressed in terms of curvature, we bypass it when we control the EPDiff in $\mathfrak{g}$ instead of controlling $\nabla_{\dot{g}(t)} \dot{g}(t)$ in $T_{g(t)} G$.

The constrained energy minimization problem for splines is,

$\underset{u}{\operatorname{minimize}}$

subject to control

subject to right action

subject to image evolution

subject to momenta duality

$$
\begin{aligned}
\frac{1}{2} \int_{0}^{1}\|u(t)\|_{\mathfrak{g}}^{2} d t & \\
u(t)-\dot{m}(t)-\operatorname{ad}_{v(t)}^{*} m(t) & =0, \\
\dot{g}(t) & =v \circ g(t), \\
I(t) & =I(0) \circ g^{-1}(t), \\
v(t) & =K \star m(t) .
\end{aligned}
$$

Similar to the Euclidean case, the Euler-Lagrange equations for the above optimization problem give an adjoint system that explains the evolution of $u$, or 
equivalently its dual, $f \in \mathfrak{g}(f=K \star u)$, such that, $\dot{f}-K \star P D I+\operatorname{ad}_{f}^{\dagger} v-\operatorname{ad}_{v} f=0$, and $\dot{P}+\nabla \cdot(P v)=0$. Here, $P$ is the adjoint variable corresponding to the image evolution constraint and the conjugate operator is, $\operatorname{ad}_{X}^{\dagger}(\cdot)=K \star \operatorname{ad}_{X} L(\cdot)$.

From Relaxation to Shooting. Notice that the above discussion is analogous to the discussion of the relaxation formulation for the Euclidean case in the sense that the Euclidean states $\left(x_{1}, x_{2}, x_{3}\right) \mapsto(g, v, f)$ in diffeomorphisms. We now convert the adjoint state, $P(t)$ to a primal state to form a forward shooting system. Analogous to the Euclidean case, this increases the order of the system by one. The shooting system for acceleration controlled motion is

$$
\begin{aligned}
\dot{g}-v \circ g & =0, & \dot{v}-f+\operatorname{ad}_{v}^{\dagger} v & =0, \\
\dot{f}-K \star P D I+\operatorname{ad}_{f}^{\dagger} v-\operatorname{ad}_{v} f & =0, & \dot{P}+\nabla \cdot(P v) & =0 .
\end{aligned}
$$

The image evolves (equivalently advects) as per the group action of $g$ on $I(0)$. Here, the vector quantity, $K \star P D I$ is analogous to $x_{4}$.

Shooting-Splines with Data-Independent Controls for Regression. Similar to the data configuration in the Euclidean example, in the context of regression, let, $J_{i}$, for $i=1 \ldots N$, denote $N$ measured images at timepoints, $t_{i} \in(0,1)$. The goal now is to define finite and relatively fewer points than the number of measurements in the interval, $(0,1)$ where $K \star P D I$ is allowed to jump. In other words, $P$ does not jump at every measurement but instead, is allowed to be free at predefined time-points that are decided independently of the data. Thus, we construct a curve $g(t)$, similar to the Euclidean case, in $G$, along the time, $t$, such that it guarantees, (a) $K \star P D I$ is not continuous, (b) $f$ is $C^{0}$-continuous, (c) $v$ is $C^{1}$-continuous, and (d) $g$ is $C^{2}$-continuous.

The unconstrained Lagrangian for spline regression takes the form:

$$
\begin{aligned}
& E\left(m^{0}, u^{0}, P^{0}, I^{0}, P^{t_{c}}, \lambda_{m}, \lambda_{u}, \lambda_{P}, \lambda_{I}\right)=\frac{1}{2 \sigma^{2}} \sum_{c=1}^{C+1} \sum_{i \in \mathcal{I}_{c}}\left\|I\left(t_{i}\right)-J_{i}\right\|^{2} \\
& +\sum_{c=1}^{C+1} \int_{0}^{1}\left\langle\lambda_{m c}, \dot{m}_{c}-u_{c}+\operatorname{ad}_{v_{c}}^{*} m_{c}\right\rangle+\left\langle\lambda_{u c}, \dot{u}-P_{c} D I_{c}+\operatorname{ad}_{f_{c}}^{*} m_{c}-K^{-1} \operatorname{ad}_{v_{c}} f_{c}\right\rangle d t \\
& +\sum_{c=1}^{C+1} \int_{0}^{1}\left\langle\lambda_{P c}, \dot{P}_{c}+\nabla \cdot\left(P_{c} v_{c}\right)\right\rangle+\left\langle\lambda_{I c}, \dot{I}_{c}+D I_{c} \cdot v_{c}\right\rangle+\left\langle\lambda_{v c}, v_{c}-K \star m_{c}\right\rangle d t \\
& +\sum_{c=1}^{C+1} \int_{0}^{1}\left\langle\lambda_{f c}, f_{c}-K \star u_{c}\right\rangle d t+\text { subject to continuity of } m, u, \text { and } I \text { at } C \text { joins. }
\end{aligned}
$$

Gradients. The optimality conditions on the gradients of the above energy functional show that the adjoint variables, $\lambda_{m}, \lambda_{u}, \lambda_{I}$, are continuous at all $C$ joins. The gradients with respect to the initial conditions are,

$$
\delta_{m_{1}^{0}} E=-\lambda_{m 1}(0), \quad \delta_{u_{1}^{0}} E=-\lambda_{u 1}(0), \quad \delta_{P_{1}^{0}} E=-\lambda_{P 1}(0), \quad \delta_{I_{1}^{0}} E=-\lambda_{I 1}(0) .
$$


We compute the gradients by integrating the adjoint system of equations within each interval backward in time,

$$
\begin{aligned}
\dot{\lambda}_{m c}+\operatorname{ad}_{\lambda_{m c}} v_{c}+\operatorname{ad}_{\lambda_{m c}}^{\dagger} v_{c}-\operatorname{ad}_{f_{c}} \lambda_{u_{c}}-\operatorname{ad}_{f_{c}}^{\dagger} \lambda_{u c}-K \star \lambda_{I c} D I_{c} & =0, \\
\dot{\lambda}_{u c}+\lambda_{m c}+\operatorname{ad}_{\lambda_{u c}}^{\dagger} v_{c}+\operatorname{ad}_{v_{c}}^{\dagger} \lambda_{u c} & =0, \\
\dot{\lambda}_{P c}+D I_{c} \cdot \lambda_{u c}+D \lambda_{P c} \cdot v_{c} & =0, \\
\dot{\lambda}_{I c}+\nabla \cdot\left(\lambda_{I c} v_{c}\right)-\nabla \cdot\left(P_{c} \lambda_{u c}\right) & =0 .
\end{aligned}
$$

All variables start from zero as their initial conditions for this backward integration. Similar to the Euclidean case, we add jumps in $\lambda_{I}$ as $\lambda_{I c}\left(t_{i}^{+}\right)-\lambda_{I c}\left(t_{i}^{-}\right)=$ $\frac{1}{\sigma^{2}}\left(I_{c}\left(t_{i}\right)-J_{i}\right)$ at measurements, $t=t_{i}$. We ensure the continuity of $\lambda_{m c}, \lambda_{u c}$, and $\lambda_{I c}$ at the joins and $\lambda_{P c}$ starts from zero at every join. We use the accumulated $\lambda_{P c+1}$ to update the jerk, $P_{c}\left(t_{k}\right)$, at the control location with,

$$
\delta_{P_{c+1\left(t_{c}\right)}\left(t_{c}\right)} E=-\lambda_{P c+1}\left(t_{c}\right) .
$$

Note this is the 'data independent' control that we motivated our formulation with. This determines the initial condition of the forward system for each interval and needs to be estimated numerically. Also note that a regularizer can be added on the initial momenta, $m_{1}^{0}$ by restricting its Sobolev norm, in which case, the gradient includes an additional term and takes the form, $\delta_{m_{1}^{0}} E=K m_{1}^{0}-\lambda_{m 1}(0)$.

\section{Results and Discussion}

We evaluate our proposed model using synthetic data and time-sequence data from the Sunnybrook cardiac MR database [8]. In these experiments, the kernel, $K$, corresponds to the invertible and self-adjoint Sobolev operator, $L=-a \nabla^{2}-$ $b \nabla(\nabla \cdot)+c$, with $a=0.01, b=0.01$, and $c=0.001$. We use fourth order RungeKutta to integrate the primal states forward and to integrate the corresponding adjoint states backwards. We use a constant stepsize gradient descent to estimate optimal initial states of spline curves and the controls. We fix the initial image, $I(0)$, and estimate initial states, $m(0), u(0), P(0)$ and $P\left(t_{c}\right)$ at control locations that completely determine the spline curve $g(t)$ from $t=0$ to $t=1$.

Experiments with Synthetic Data. To assess the strength of spline regression on non-geodesic image data, we create a synthetic sequence of $N=10$ shapes to simulate non-monotonic dynamics from $t=0$ to $t=1$ (Fig. 2, first row). The synthetic shape expands till $t=0.2$ and then contracts till $t=0.7$ and finally expands again till it reaches the end point, at $t=1.0$. Using such data, we attempt to simulate an inflection point in the velocity or the rate of shape change at $t=0.5$. We observe that adding a single spline control (second row) in the mid point results in the best fit that summarizes the smooth dynamics of change. The reported error of fit corresponds to $L^{2}$ image residual as per the data-likelihood in Eq. (5). The estimated diffeomorphism successfully captures the initial trend of expanding shape, followed by its contraction and finally culminating in expansion. Without adding any control, the resulting spline 


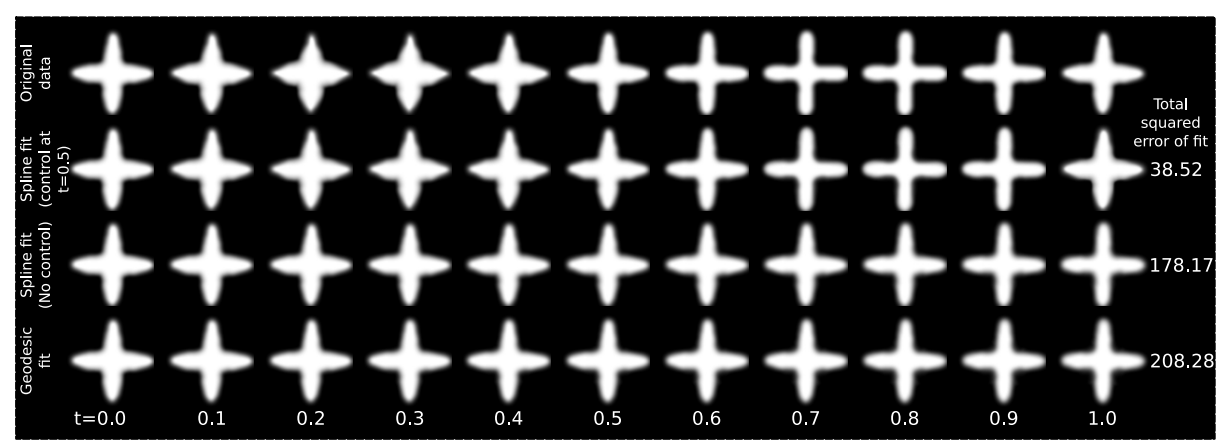

Fig. 2. Comparison of spline regression models: identical stepsizes and number of iterations of gradient descent were used for all models on synthetic data. More information about all estimated states and video visualizations are in the supplementary material.

trend, even though non-monotonic, exhibits a less flexible dynamics and fails to recover the inflection point in the rate of shape change. Finally, being the most inflexible, geodesic regression performs worst, and barely captures any real spatio-temporal trend.

Experiments with Cardiac Data. The cardiac time-sequence data corresponds to 20 snapshots at equal intervals of the beating heart of a normal individual with age $=63$ years (Subject Id: SCD0003701). We cropped all the axial images to a common rectangular region around the heart followed by histogram matching to align intensities of all the timepoints to the image at $t=0$. Fig. 3 . shows the original scans (first row) and the result of regression models (second to fourth row). We only display half of the timepoints than those actually used to fit the model. Similar to the synthetic data, we observe that the original data exhibit a non-geodesic and non-monotonic trend in changing shape of the beating heart. The comparison in terms of the error of fit for all models suggests that both spline curves perform better than the geodesic. In other words, we obtained a slightly better fit for the spline curve with single control when compared to the spline curve without any control. The geodesic fit again performs worst out of the three models. The dynamics of the beating heart for these models are best seen in the multimedia file in the supplementary material. 1

We stress that in all our experiments, the full diffeomorphic paths and the evolution of all states along the estimated curves are completely parameterized by very few parameters ( 4 for spline fit with 1 control and 3 for spline fit without any control) that are independent of the study size. The estimated models are expected to fit better with increasing number of control points, which will necessitate model selection methods. Due to the limitations of our current optimization method, i.e., the constant stepsize gradient descent, we did not try

${ }^{1}$ Supplementary material including derivations, video visualizations, and the CPU/GPU implementations of the shooting splines for 2D and 3D image sequences is at https://nikhilsingh@bitbucket.org/nikhilsingh/diffeosplines.git 


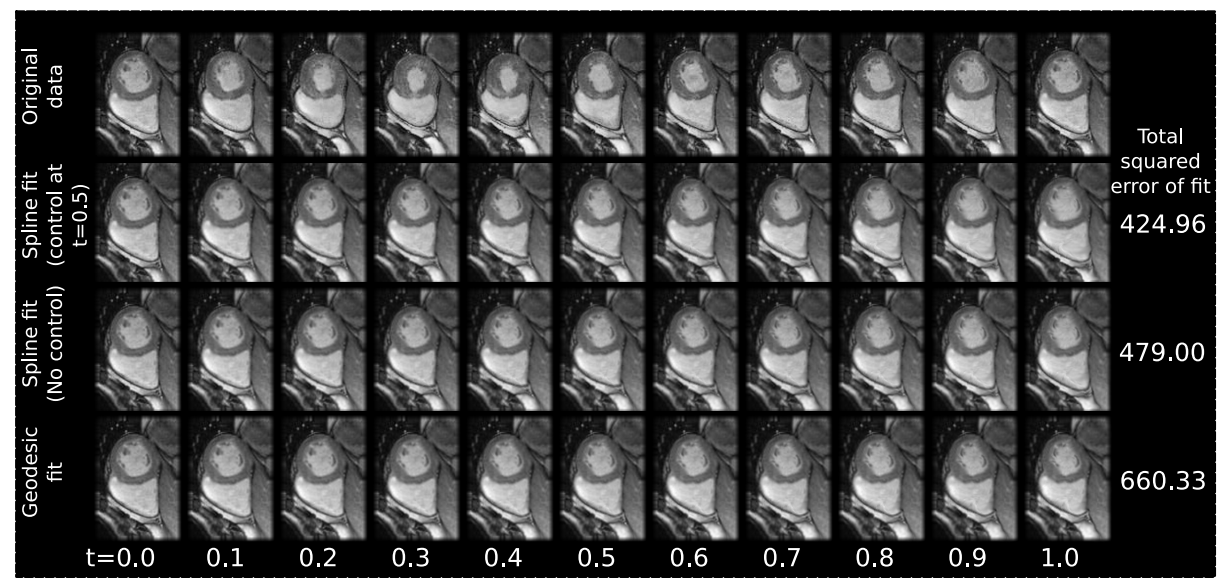

Fig. 3. Spline regression models on cardiac MRI breathing data. Comparison is better seen in the video visualizations in supplementary material

spline estimations with more than one control point. This will be the subject of future work. Another aspect could be investigating possibilities of combining our model on diffeomorphisms with the higher order models on shapes 411]. This method of shooting splines in diffeomorphisms lays a foundation to model flexible dynamics of shape changes seen in time series of medical images, and in particular, opens the possibility to model periodic data by adding periodicity constraints.

Acknowledgments. This research is supported by grants, NSF EECS-1148870, NSF EECS-0925875, and NIH R01-MH091645.

\section{References}

1. Arnol'd, V.I.: Sur la géométrie différentielle des groupes de Lie de dimension infinie et ses applications à l'hydrodynamique. Ann. Inst. Fourier 16, 319-361 (1966) 124

2. Bruveris, M., Gay-Balmaz, F., Holm, D., Ratiu, T.: The momentum map representation of images. Journal of Nonlinear Science 21(1), 115-150 (2011) 124

3. Camarinha, M., Leite, F.S., Crouch, P.: Splines of class ck on non-euclidean spaces. IMA Journal of Mathematical Control and Information 12(4), 399-410 (1995) 124

4. Gay-Balmaz, F., Holm, D.D., Meier, D.M., Ratiu, T.S., Vialard, F.X.: Invariant higher-order variational problems II. J. Nonlinear Science 22(4), 553-597 (2012) 128

5. Hinkle, J., Fletcher, P., Joshi, S.: Intrinsic polynomials for regression on Riemannian manifolds. Journal of Mathematical Imaging and Vision, 1-21 (2014) 121

6. Niethammer, M., Huang, Y., Vialard, F.-X.: Geodesic regression for image timeseries. In: Fichtinger, G., Martel, A., Peters, T. (eds.) MICCAI 2011, Part II. LNCS, vol. 6892, pp. 655-662. Springer, Heidelberg (2011) 121 
7. Noakes, L., Heinzinger, G., Paden, B.: Cubic splines on curved spaces. IMA Journal of Mathematical Control and Information 6(4), 465-473 (1989) 124

8. Radau, P., Lu, Y., Connelly, K., Paul, G., Dick, A., Wright, G.: Evaluation framework for algorithms segmenting short axis cardiac MRI (July 2009) 126

9. Singh, N., Hinkle, J., Joshi, S., Fletcher, P.: A vector momenta formulation of diffeomorphisms for improved geodesic regression and atlas construction. In: ISBI, pp. 1219-1222 (2013) 121

10. Singh, N., Hinkle, J., Joshi, S., Fletcher, P.T.: A hierarchical geodesic model for diffeomorphic longitudinal shape analysis. In: Gee, J.C., Joshi, S., Pohl, K.M., Wells, W.M., Zöllei, L. (eds.) IPMI 2013. LNCS, vol. 7917, pp. 560-571. Springer, Heidelberg (2013) 121

11. Trouvé, A., Vialard, F.X.: Shape splines and stochastic shape evolutions: A second order point of view. Quarterly of Applied Mathematics 70(2), 219-251 (2012) 121 124, 128

12. Younes, L.: Shapes and Diffeomorphisms, vol. 171. Springer, Berlin (2010) 124

13. Younes, L., Arrate, F., Miller, M.I.: Evolution equations in computational anatomy. NeuroImage 45(1, suppl.), S40-S50 (2009) 124 